\title{
A WSN for Ground-level Ozone Monitoring based on Plant Electrical Activity Analysis
}

\author{
S. Morosi*, M. Dolfi*, E. Del Re*, E. Masi ${ }^{\dagger}$, I. Colzi $^{\dagger}$, S. Mancuso ${ }^{\dagger}$, F. Francini ${ }^{\ddagger}$, and A. Valgimigli ${ }^{\ddagger}$ \\ ${ }^{*}$ CNIT - DINFO, University of Florence, via S. Marta 3, 50139 Florence, Italy \\ Email: marco.dolfi@unifi.it, simone.morosi@unifi.it, enrico.delre@unifi.it \\ ${ }^{\dagger}$ DISPAA, University of Florence, viale delle Idee 30, 50019 Sesto Fiorentino, Florence, Italy \\ Email: ilaria.colzi@unifi.it, elisa.masi@unifi.it, stefano.mancuso@unifi.it \\ ${ }^{\ddagger}$ ETG s.r.l., Via di Porto 159, 50018 Scandicci, Florence, Italy \\ Email: f.francini@etgsrl.it, ingegneria@etgsrl.it
}

\begin{abstract}
This paper describes an innovative monitoring technology for detecting ground-level ozone pollution, b ased on the deployment of a network of wireless devices connected to a collection of plants, used as bio-sensors. Such devices retrieve and transmit the electrical activity signals experienced in plants, used to monitor environmental conditions. The distributed plants as sensors infrastructure communicates wirelessly with a weather station, equipped with meteorological sensors as well as data logging and both wireless and wired communication capabilities. A back end server collects and analyze real-time data from the station and delivers environmental and ozone pollution information to users through a web portal. In order to perform a classification on the level of ozone exposure, a correlation-based approach has been used. The system is implemented in pilot phase with a sensing infrastructure of four plants and started operation in November 2014. As the data collection volume increases, more accurate classification techniques can be performed.
\end{abstract}

\section{INTRODUCTION}

Nowadays, the air quality is getting worse in highly anthropized environments: this phenomenon stimulates a high level of interest within the scientific community and public opinion because of the known strong relationship between exposure to many air pollutants and increased adverse effects on the human health [1]-[3]. Weather monitoring is also important not just in defining present climate, but also for detecting changes in climate and providing the data inputs to the models which enable us to predict future changes in our environment [4], [5]. Because of the wide variety of uses for the information, different groups of people are interested in a subset of a large number of environmental variables. These include solar radiation, wind speed, wind direction, barometric pressure, air temperature, humidity and net radiation. The demand for these data, usually on an hourly or more frequent timescale, has increasingly been met by the development and widespread deployment of automatic weather stations (AWSs) over the past 30 years [6]. Developments in communication technologies allow more remote, real-time weather monitoring and access. Recently there has been a very fast growth in the number of the active weather stations and a great improvement of their communication abilities, which has helped to facilitate access to weather information from a limited number of stations run by the operator, to a wider number of networks of stations. The availability of data from spatially distributed networks has resulted in a paradigm shift, namely from considering the weather at a single point towards the evolution of weather patterns over large areas. Moreover, the new generation of weather sensors should be less sensitive to chemical interference, and have improved long term stability and negligible hysteresis. This trend should continue since new innovative sensors are likely to appear: particularly, visibility monitors are increasingly common on road monitoring stations and the upcoming monitoring chemical variables will operate not only in the atmosphere (e.g. ozone, $\mathrm{SO}_{2}$ and other industrial pollutants), but also in rainfall and even in the soil.

The monitoring of current near-surface atmospheric conditions with a weather station can give us important local information, but little about how those conditions arose. An important control on those conditions is the flux of water and energy, because a wide range of organic and inorganic gaseous pollutants are released into the atmosphere. Among air pollutants, ozone is one of the most important greenhouse gas [7] with secondary origin, generated in the troposphere through a series of complex photochemical reactions involving solar radiation and ozone precursors, i.e. methane $\left(\mathrm{CH}_{4}\right)$, carbon monoxide (CO), volatile organic compounds (VOCs), and nitrogen oxides $\left(\mathrm{NO}_{\mathrm{x}}\right)$, which are largely emitted from anthropogenic sources [8]. Background $\mathrm{O}_{3}$ concentrations have risen from $\sim 10 \mathrm{ppb}$ before the industrial revolution [9] to daytime summer concentrations exceeding $40 \mathrm{ppb}$ in many parts of the Northern Hemisphere [10]. Due to its nature of reactive oxidant agent, ozone can generate several negative effects on human health including lung inflammation, reduced lung function, degenerative diseases, age related disorders and eventually cancer [11]. Ozone also acts as a corrosive agent for many materials, surface coatings and buildings [12].

The use of plants as biosensors represents a new reliable approach for ozone monitoring. In comparison with the traditional monitoring systems, the use of biosensors has the advantage to show us the real impact of pollutants on living organisms, thus providing additional data to the electronic instruments. Moreover, this allows to take into account the concepts of bioavailability, dose and exposure, resulting in a more realistic approach of the impact of pollutants on environmental and human health [13]. Plants are more sensitive than humans and animals in terms of physiological reaction to fluctuations of multiple parameters [14], making them suitable tools for environmental monitoring. More specifically, morphological and anatomical parameters of plants, such as specific leaf area (SLA), stomatal density (SD), and pore surface, have proven to be useful indicators of air quality [15]. The observation of 
necrosis on leaves and coloured spots allow, in certain cases, the identification of pollutant sources.

In this paper, an ozone pollution monitoring system based on plant electrical activity analysis is presented. An automatic weather station provides crucial data about changes to the composition of the atmosphere, gathering site-specific, realtime data on environmental conditions such as wind speed and direction, temperature, barometric pressure and precipitation. The station uses reliable and accurate sensors to measure these and other ambient weather conditions. These parameters provide insight into bio-meteorological problems, which may relate to food production, human health, protection of endangered flora and fauna or ensuring a comfortable environment for habitation. The data collected is also used to warn the public about hazardous air quality; as a matter of fact the system can be accessed in real-time by authorized users via web applications. The ground-level ozone monitoring system is based on data coming from a network of plants, which share information about the environment, and on a data processing platform located on a back end server, where a classification algorithm able to identify the plant response to critical level of ozone concentrations is adopted. As a result, the proposed platform uses the plant community as a distributed, eco-compatible, self-sustainable and cost-effective sensing infrastructure.

The paper is organized as follows: in Section II, a description of the system is given. The ozone critical level detection technique based on plant electrical activity analysis is presented in Section III. Conclusions are drawn in Section IV.

\section{SYSTEM DESCRIPTION}

The system architecture for ground-level ozone monitoring is shown in Figure 1. The deployed network is composed by four plant sensors, which have been actively operating since November 2014: the current deployment is a pilot test and more types of performance assessment and improvement measures are planned in the future. The air monitoring system consists of two main subsystems: the distributed plant sensing infrastructure, and the automatic weather station. The air monitoring station communicates with a back end server, where a data processing platform is responsible for the interpretation of the plant signals in order to evaluate critical levels of ozone concentrations. To display the collected and processed information in a user-friendly format, a web platform accessible by registered users has been developed.

More detailed description of these subsystems is given in the following subsections.

\section{A. Plants as Biosensors Infrastructure}

About $50 \mathrm{~cm}$ high plants of Ligustrum texanum and Buxus macrophilla were deployed in a delimited area. Electrical signals are monitored by means of three stainless steel needle electrodes, one placed at the base (reference for background noise subtraction), one in the middle and the other on top of the stem. The electrodes have isolated cable and the needles are $0.45 \mathrm{~mm}$ in diameter and $25 \mathrm{~mm}$ in length, similar to those used in electromyography. The electrodes are inserted around $57 \mathrm{~mm}$ into the plantstem so that the sensitive active part of the electrodes $(2 \mathrm{~mm})$ are in contact with the plant cells. After some preliminary tests, the sampling frequency was set

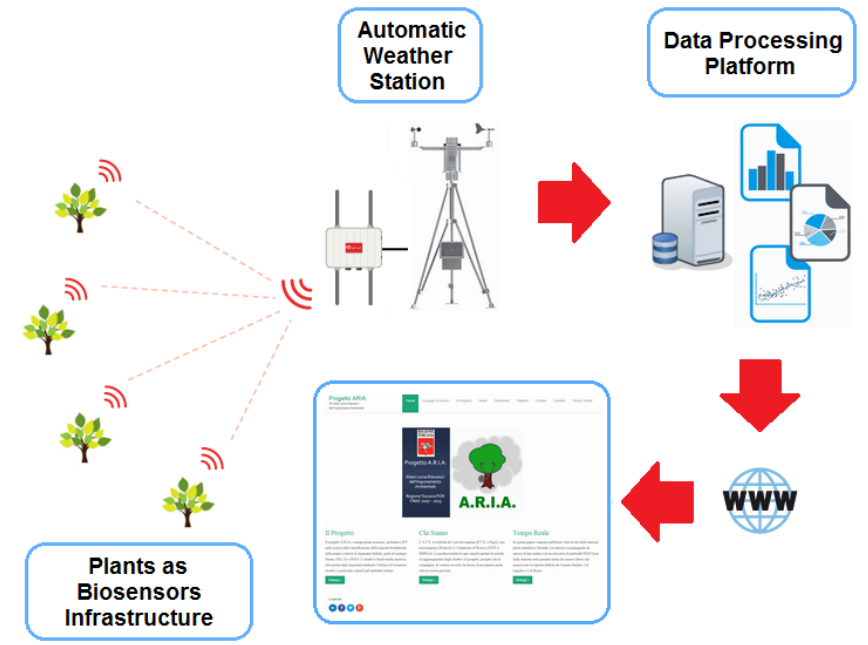

Fig. 1. Ground-level Ozone Monitoring System Architecture.

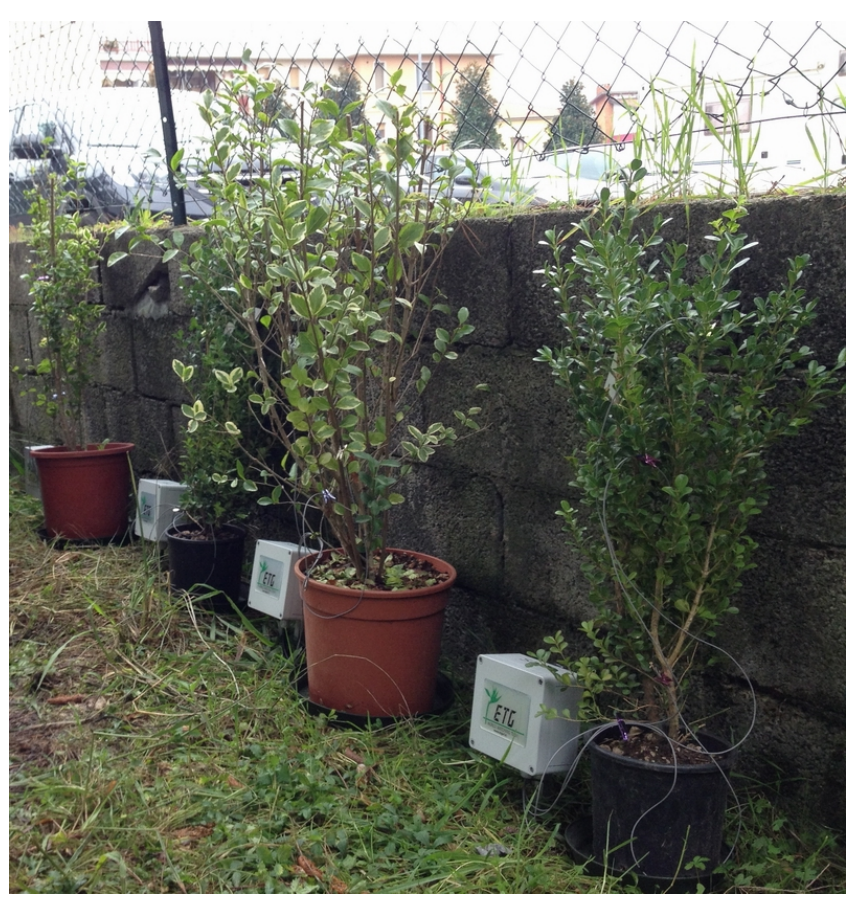

Fig. 2. The deployed ligustrum and buxus plants as biosensors.

as $1 \mathrm{sample} / \mathrm{s}$ for all the deployed sensors. All the sensing elements are connected to a data logging and communication board, equipped with Freescale iMx28 processor and external MicroSD memory with $1 \mathrm{~GB}$ capacity. Its main functions are data acquiring, processing, logging and transmitting. Each device houses a Wi-Fi modem for wireless connectivity to the weather station board, which establishes a TCP/IP Internet connection with the data processing platform located at the back end server. A picture of the deployed monitoring sensors is shown in Figure 2.

\section{B. Automatic Weather Station}

The automatic weather station consists of several meteorological sensing elements, the data logging and wireless 


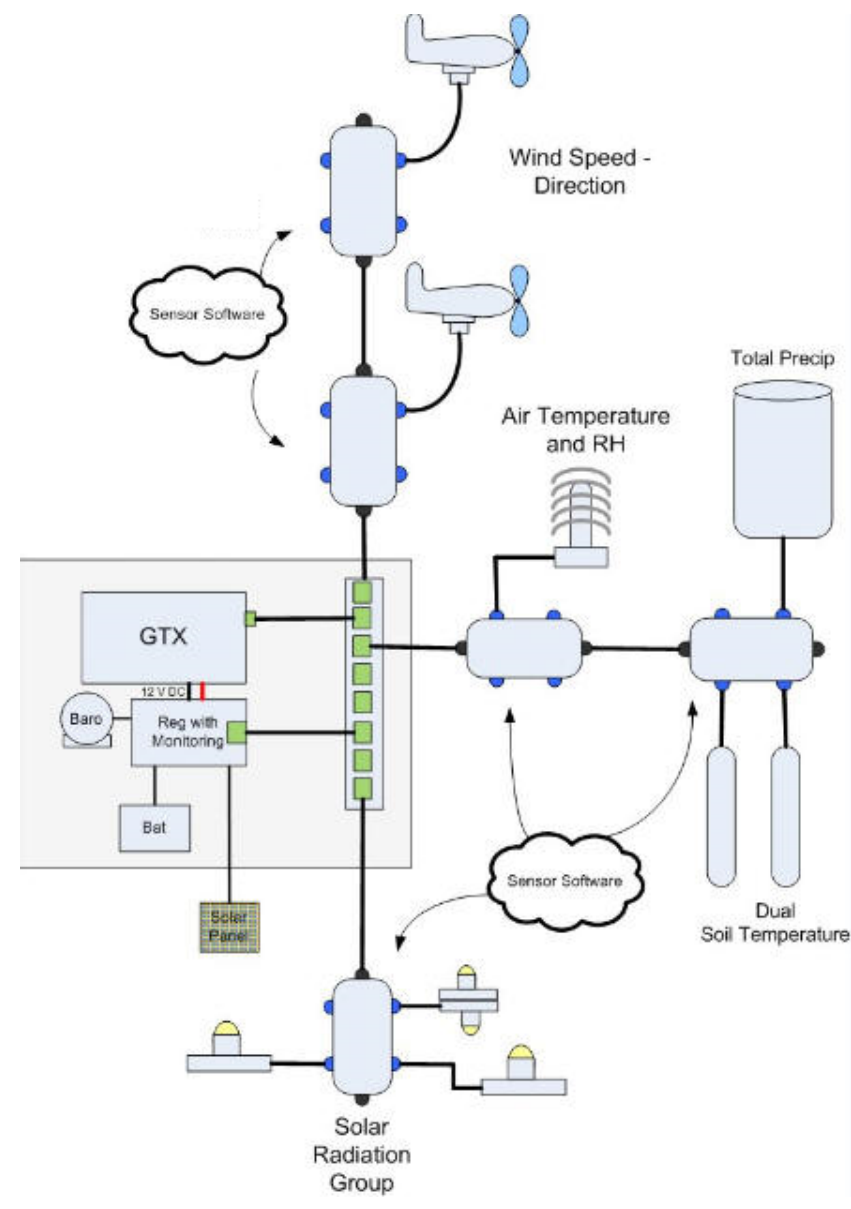

Fig. 3. AWS solution scheme with integrated sensing elements.

communication board, and the power supply system. When studying air quality, it is important to measure the following factors, as they can help understand the chemical reactions that occur in the atmosphere: wind speed and direction, temperature, humidity, rainfall, solar radiation. An example scheme of automatic weather station with full integration of meteorological sensing components is depicted in Figure 3. When high pollutant concentrations occur at a monitoring station, wind data records can determine the general direction and area of the emissions. The identification of the sources can allow to plan a reduction of the impacts on air quality.

Measuring temperature supports air quality assessment, air quality modeling and forecasting activities. Temperature and sunlight (solar radiation) play an important role in the chemical reactions that occur in the atmosphere to form photochemical smog from other pollutants: favourable conditions can lead to increased concentrations of smog.

Like temperature and solar radiation, water vapour plays an important role in many thermal and photochemical reactions in the atmosphere. As water molecules are small and highly polar, they can bind strongly to many substances: if attached to the particles which are suspended in the air they can significantly increase the amount of light scattered by the particles (monitoring aerosols). If the water molecules attach to the corrosive gases, such as sulfur dioxide, the gas will dissolve in the water and form an acid solution that can damage health and property. Reporting of the water vapour content of air gives information

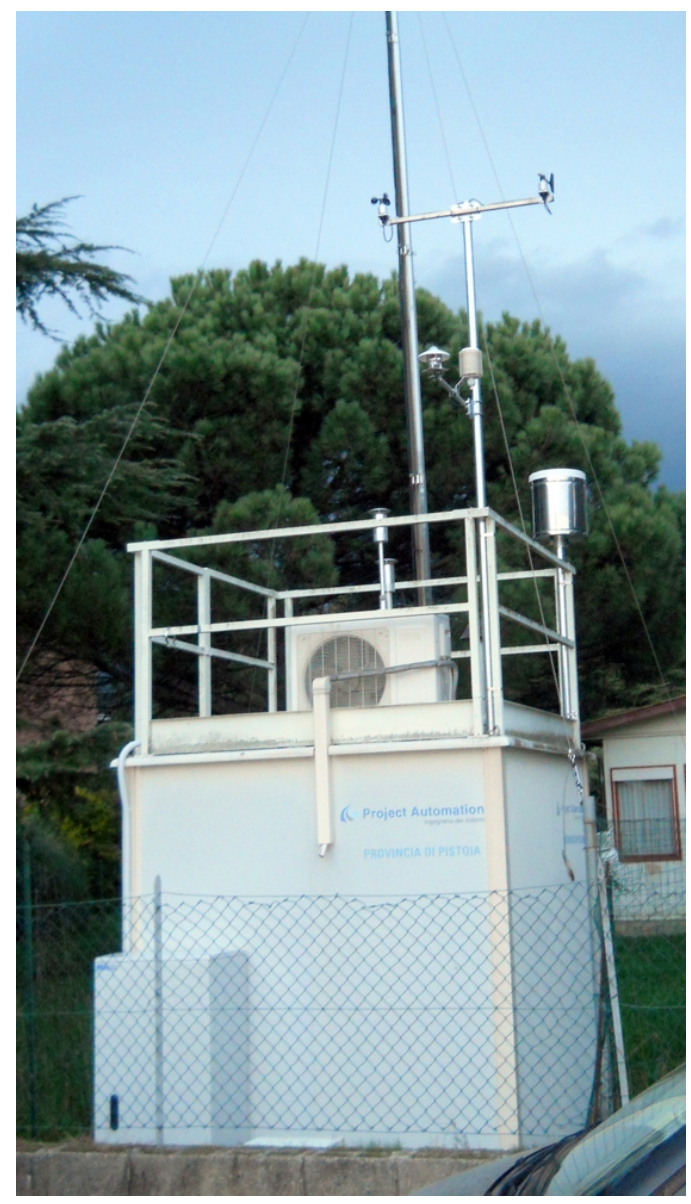

Fig. 4. The automatic weather station equipped with meteorological sensors.

about the percentage of the saturation vapour pressure of water at a given temperature, namely about the relative humidity. The amount of water vapour in the atmosphere is highly variable: it depends on geographic location, how close water bodies are, wind direction and air temperature. Relative humidity is generally higher during summer when temperature and rainfall are also at their highest.

Rain has a 'scavenging' effect when it washes particulate matter out of the atmosphere and dissolves gaseous pollutants. Removing particles improves visibility. Where there is frequent high rainfall, air quality is generally better. If the rain dissolves gaseous pollutants, such as sulfur dioxide, it can form acid rain resulting in potential damage to materials or vegetation. A common method to measure rainfall is to use a tipping bucket rain gauge, which registers rainfall by counting small amounts of collected rain. All the sensing elements of the weather station are connected to the analog inputs of the data logging and communication board, equipped with Linux-based embedded operating system and SQLite database to provide efficient and reliable local data storage. The data logger is equipped with a GPRS modem for wireless connectivity and with Ethernet networking interface to establish a TCP/IP Internet connection with the data processing platform located at the back end server. A picture of the deployed weather station is shown in Figure 4. 


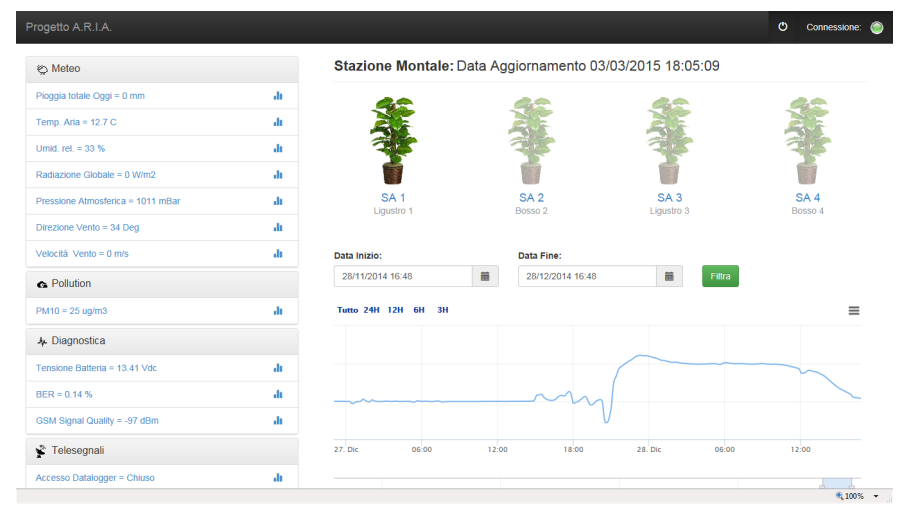

Fig. 5. Plant electrical signal measurements view.

\section{Client-side Web Applications}

The dissemination of the measured environmental conditions is performed via client-side web applications accessible through any browser, or any computer or mobile device. The stored data received from the sensors are forwarded by the server to the applications that access the network. These applications, hosted on a web platform, provide updated data summaries and statistics, data visualization with display of real-time sensors measurements, including plants electrical activity signals, and data dissemination with weather warnings relating to detected critical levels of ozone pollution in the area. The web application for the described end-to-end system, named ARIA (Italian acronym for "plants as detectors of environmental pollution"), is available at the following link: http://www.progettoaria.net/. Although the system is in pilot phase, the sensors measurements are already accessible after registering to the site. Among other applications, the website allows displaying the plants electrical signals and by clicking on a particular sensor icon the most recent measurements at the plant location is shown. A web view example is illustrated in Figure 5. In addition, detailed reports about the detected ozone pollution levels are provided. When the plants electrical response to ozone exposure reaches warning levels, a visual signal alert is displayed on the website.

In the next section, the ground-level ozone pollution detection system based on plant electrical activity analysis is presented.

\section{OZONE CRITICAL LEVEL DETECTION}

The data processing platform is operating on a back end server. The focus at the preliminary stage of this subsystem was to find an association between ozone exposure and some typical features in the resulting plant electrical signal, developing a classification algorithm able to identify the stimulus. In order to obtain reliable results, automatic response detection and data classification for plant electrical signals were to be implemented. Many studies reported artifacts detection methods for EEG and EKG analysis [16]-[20]. These methods were appropriate for human biological signals and offline analysis. For the analysis of plants bio-electrical signals that are related to environmental and climatic changes the response detection algorithm needs to be simplified. The detection algorithm of plant response to ozone is based on a preliminary extraction of significant deviations from a certain baseline trend: in order

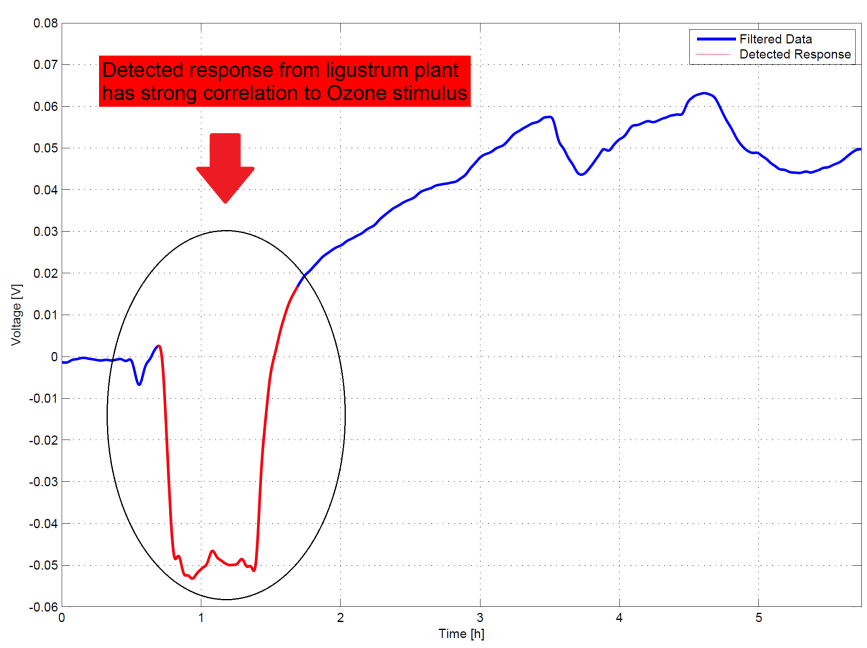

Fig. 6. Detection of critical level of ozone exposure for ligustrum plant sensor.

to correctly identify the response in an automatic way, a derivative-based algorithm has been used, similarly to those used in spike detection [21]. Subsequently the classification of the ozone risk level is developed by using the method of correlation. A representative ozone response template, constructed by coherent averaging of the respective response segments of the recordings used for the preliminary training phase, was employed for subsequent comparison with all the responses detected by the implemented system.

Cross correlation is a statistical technique which can show whether and how strongly pairs of variables are related. It is an excellent tool to match images and signals with each other. It is robust to noise, and can be normalized for pattern matching. The correlation coefficient, a statistical measure of similarity of two waveforms, produces a value, $\rho$, which falls within the range $[-1,+1]$, where +1 indicates a perfect match between signal and template. Mathematically, the correlation coefficient is defined as follows:

$$
\rho=\frac{\sum_{i=1}^{N}\left(t_{i}-\bar{t}\right)\left(s_{i}-\bar{s}\right)}{\sqrt{\sum_{i=1}^{N}\left(t_{i}-\bar{t}\right)^{2}} \sqrt{\sum_{i=1}^{N}\left(s_{i}-\bar{s}\right)^{2}}}
$$

where $t_{i}$ are the template points, $s_{i}$ are the signal points under analysis, $\bar{t}$ is the average value of the template points, $\bar{s}$ is the average value of the signal points, $N$ is the number of points in the template, and $\rho$ is the performance measure. The correlation coefficient is independent of the relative amplitudes of two signals and independent of any baseline changes. Based on experimental data, it was observed that the plant response to ozone stimulus is characterized by a specific waveform. The automatic detection system takes advantage of this property to classify the risk level of ozone air pollution by using the correlation coefficient. An example of detected ozone response is depicted in Figure 6.

\section{CONCLusions}

In this paper an end-to-end system for ground-level ozone monitoring based on plants employed as biosensors is presented. The system has two main components: the plants as 
biosensors infrastructure representing a wireless sensor network for ozone exposure detection, and the automatic weather station equipped with fundamental meteorological sensors. The monitoring station communicates with a back end server using GPRS or Ethernet communications. The data processing platform hosted at the back end server is able to identify critical levels of ozone exposure by applying a correlation-based classification algorithm. A web platform has been developed to allow registered users to access both historical and realtime data. The current system is in pilot phase and additional techniques under current investigation include the extension of the developed algorithms based on plant electrical activity analysis for further classification of other major air pollutant classes.

\section{REFERENCES}

[1] Robert L. Maynard, "Chapter 6 Health Effects of Urban Pollution," in Air Quality in Urban Environments, vol. 28, pp. 108-128. The Royal Society of Chemistry, 2009.

[2] Robert J. Laumbach and Howard M. Kipen, "Respiratory health effects of air pollution: Update on biomass smoke and traffic pollution," Journal of Allergy and Clinical Immunology, vol. 129, no. 1, pp. 3 11, 2012.

[3] Mauro Masiol, Claudio Agostinelli, Gianni Formenton, Enzo Tarabotti, and Bruno Pavoni, "Thirteen years of air pollution hourly monitoring in a large city: potential sources, trends, cycles and effects of car-free days," The Science of the total environment, vol. 494-495, pp. 8496, October 2014.

[4] J.F. Artiola, I.L. Pepper, and M.L. Brusseau, Environmental Monitoring and Characterization, Elsevier Academic Press, 2004.

[5] Matthew Hobby, Matthew Gascoyne, John H. Marsham, Mark Bart, Christopher Allen, Sebastian Engelstaedter, Dieh Mohamed Fadel, Abdoulaye Gandega, Richard Lane, James B. McQuaid, Bouziane Ouchene, Abdelkader Ouladichir, Douglas J. Parker, Phil Rosenberg, Mohammed Salah Ferroudj, Azzedine Saci, Fouad Seddik, Martin Todd, Dan Walker, , and Richard Washington, "The fennec automatic weather station (aws) network - monitoring the saharan climate system," $J$. Atmos. Oceanic Technol., vol. 30, pp. 709724, 2013.

[6] Wmo, Guide to Meteorological Instruments and Methods of Observation, World Meteorological Organization, 7 bis, avenue de la Paix, P.O. Box No. 2300, CH-1211 Geneva 2, Switzerland, seventh edition, 2008.

[7] Arlene M. Fiore, Daniel J. Jacob, Brendan D. Field, David G. Streets, Suneeta D. Fernandes, and Carey Jang, "Linking ozone pollution and climate change: The case for controlling methane," Geophysical Research Letters, vol. 29, no. 19, pp. 25-1-25-4, 2002.

[8] Elena Paoletti, Alessandra De Marco, David C.S. Beddows, Roy M. Harrison, and William J. Manning, "Ozone levels in European and USA cities are increasing more than at rural sites, while peak values are decreasing," Environmental Pollution, vol. 192, no. 0, pp. 295 299, 2014.

[9] A. VOLZ and D. KLEY, Evaluation of the Montsouris series of ozone measurements made in the nineteenth century, 1988.

[10] R. Vingarzan, "A review of surface ozone background levels and trends," Atmospheric Environment, vol. 38, pp. 3431-3442, 2004.

[11] Marilena Kampa and Elias Castanas, "Human health effects of air pollution," Environmental Pollution, vol. 151, no. 2, pp. 362 - 367 , 2008, Proceedings of the 4th International Workshop on Biomonitoring of Atmospheric Pollution (With Emphasis on Trace Elements).

[12] M. Escudero, A. Lozano, J. Hierro, J. d. Valle, and E. Mantilla, "Urban influence on increasing ozone concentrations in a characteristic Mediterranean agglomeration," Atmospheric Environment, vol. 99, pp. 322-332, Dec. 2014.

[13] Jaro Falla, Philippe Laval-Gilly, Michel Henryon, Dominique Morlot, and Jean-franois Ferard, "Biological Air Quality Monitoring: a Review," Environmental Monitoring and Assessment, vol. 64, no. 3, pp. 627-644, 2000
[14] Tatiana Wuytack, Kris Verheyen, Karen Wuyts, Fatemeh Kardel, Sandy Adriaenssens, and Roeland Samson, "The potential of biomonitoring of air quality using leaf characteristics of white willow (Salix alba L.)," Environmental Monitoring and Assessment, vol. 171, no. 1-4, pp. 197204, 2010.

[15] B.L.W.K. Balasooriya, R. Samson, F. Mbikwa, U.W.A. Vitharana, P. Boeckx, and M. Van Meirvenne, "Biomonitoring of urban habitat quality by anatomical and chemical leaf characteristics," Environmental and Experimental Botany, vol. 65, no. 23, pp. 386 - 394, 2009.

[16] JunFeng Gao, Yong Yang, Pan Lin, Pei Wang, and ChongXun Zheng, "Automatic Removal of Eye-Movement and Blink Artifacts from EEG Signals," Brain Topography, vol. 23, no. 1, pp. 105-114, 2010.

[17] P. LeVan, E. Urrestarazu, and J. Gotman, "A system for automatic artifact removal in ictal scalp EEG based on independent component analysis and Bayesian classification," Clinical Neurophysiology, vol. 117, no. 4, pp. 912 - 927, 2006.

[18] Arnaud Delorme, Terrence Sejnowski, and Scott Makeig, "Enhanced detection of artifacts in EEG data using higher-order statistics and independent component analysis," NeuroImage, vol. 34, no. 4, pp. 1443 - 1449, 2007.

[19] Joe-Air Jiang, Chih-Feng Chao, Ming-Jang Chiu, Ren-Guey Lee, Chwan-Lu Tseng, and Robert Lin, "An automatic analysis method for detecting and eliminating ECG artifacts in EEG," Computers in Biology and Medicine, vol. 37, no. 11, pp. 1660 - 1671, 2007.

[20] Maarten van de Velde, I.Robert Ghosh, and Pierre J.M. Cluitmans, "Context related artefact detection in prolonged EEG recordings," Computer Methods and Programs in Biomedicine, vol. 60, no. 3, pp. 183 - 196, 1999.

[21] Scott B. Wilson and Ronald Emerson, "Spike detection: a review and comparison of algorithms," Clinical Neurophysiology, vol. 113, no. 12, pp. $1873-1881,2002$. 